LECTURE

\title{
Circuit Theory Based on New Concepts and Its Application to Quantum Theory
}

\section{Reconsideration of Maxwell Equation for Application of Quantum Theory}

\author{
Nobuo Nagai ${ }^{1}$ (Hokkaido University), Hirofumi Sanada ${ }^{2}$ (Hokkaido University of Science) \\ and Takashi Yahagi ${ }^{3}$ (Signal Processing Technology Laboratory) \\ E-mail:1nagai@es.hokudai.ac.jp, 2sanada@hus.ac.jp, 3yahagi@risp.jp
}

\begin{abstract}
Coils and capacitors are circuit elements that induce magnetic and electric fields, which are the fields of reactive power, when they are used independently. When multiple (n) LC ladder circuits are constructed using coils and capacitors and periodically connected, a transmission circuit that transmits active power is obtained. Because this circuit induces the phase $\mathrm{k} \pi(\mathrm{k}=1,2, \cdots, \mathrm{n}-1)$, the resonance angular frequency is obtained. Moreover, we examine a method of analytical mechanics for analyzing the Maxwell equations using a Hamiltonian, in which the path of light is examined considering refraction. The refraction of light waves means the generation of reflected waves, but the energy of the reflected waves is not considered in this method. Thus, it is shown that the analytical method does not satisfy the law of conservation of energy.
\end{abstract}

Keywords: LC ladder circuit, reactive and active powers, periodic structure, image and iterative parameters, resonant phase $\mathrm{k} \pi$, Hamiltonian, energy conservative law, variational calculus, Fermat's principle, refraction, reflection

\section{Introduction}

In Session 21, we showed a method of applying analog circuit theory to the Maxwell equations using telegrapher's equations. It is said that the discovery of the Maxwell equations induced a paradigm shift in science and opened a way to today's advanced research including the theory of relativity and quantum mechanics [1]. In this session, we analyze the Maxwell equations by applying analog circuit theory, which is different from a method of analyzing hyperbolic partial differential equations conventionally used in mathematics [2], and reexamine the key features of the Maxwell equations.

In physics, waves are expressed by real trigonometric functions because the vibration of strings satisfies a wave equation. In quantum mechanics, the Schrödinger equation is a wave equation expressed by complex functions, and hence, complex numbers are required for the wave equation in quantum mechanics. In circuit theory, Heaviside demonstrated that complex numbers are required to express waves by considering the operational calculus and telegrapher's equations, and clearly showed that reactive power exists in lossless circuits and in the steady state, as shown in Session 21. Here, the phenomena involving some actions in analog circuit theory (phenomena related to time t) are transient phenomena, but transient responses are excluded from our discussion because they include difficult problems.

In this session, we examine the physical properties of waves obtained using complex numbers for voltage and current in the steady state.

\section{Complex Impedance in Steady State}

Conventionally, the Maxwell equations are analyzed by three-dimensional vector analysis (a method of analytical mechanics). Here, we analyze the Maxwell equations by applying circuit theory. This method is one-dimensional analysis but can determine the steady-state response. The lossless properties and reactive power can be obtained using complex numbers, and their use is discussed below.

A wave equation can be obtained from the Maxwell equations. As shown in Session 19, an LC ladder circuit can be obtained by quantizing a wave equation. Here, we 
examine the roles of a coil and a capacitor that constitute an LC ladder circuit as well as its periodicity to make effective use of the roles.

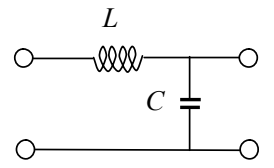

(a)

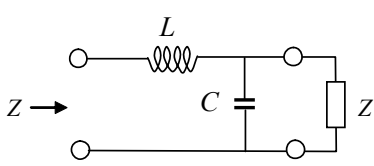

(b)
Fig.22.1 (a) Diagram and (b) impedance representation of an asymmetric LC ladder circuit.

Figure 22.1(a) shows an LC ladder circuit, which was described in Session 5. Here, we examine the effect of using complex numbers.

\subsection{Properties of coils and capacitors}

In a textbook of physical mathematics [3] used in physics, coils and capacitors are explained in the section on electric vibration, but their features obtained using complex numbers are not described. Here, we examine the features of coils and capacitors expressed using complex numbers.

The relationship between voltage and current for a coil with inductance $L$ and a capacitor with capacitance $C$, respectively, in the steady state are given by

$$
\begin{aligned}
& V=j \omega L \cdot I \\
& I=j \omega C \cdot V
\end{aligned}
$$

Their phase differences between voltage and current are both $\pi / 2$ and they are perpendicular. The powers as a product of voltage and current are given by imaginary numbers, meaning that it is reactive power.

Thus, coils and capacitors are lossless circuit elements when used independently because reactive power given by an imaginary number is obtained. Therefore, for a circuit where finite arbitrary numbers of coils and capacitors are connected, the impedance is still given by a purely imaginary number, and the reactive power is obtained.

As described in Session 5, the input impedance of an infinitely repeated circuit, $Z$, is unchanged even when one more segment is added. Such a circuit is expressed in Fig. 22.1(b) and $Z$ is given by

$$
\begin{gathered}
Z=j \omega L+\frac{1}{j \omega C+1 / Z} \\
\therefore j \omega C Z^{2}+\omega^{2} L C Z-j \omega L=0
\end{gathered}
$$

Equation (22.3b) is a quadratic equation. The solution of the equation, $Z$, has a real part when the following condition holds.

$$
0 \leqq \omega<\frac{2}{\sqrt{L C}} \equiv \omega_{c}
$$

Here, the upper limit of the angular frequency $\omega_{c}$ is an important quantity, as explained later, and is called the cutoff angular frequency.

When Eq. (22.4) holds, $Z$ in Eq. (22.3b) is given by

$$
Z= \pm \sqrt{\frac{L}{C}} \sqrt{1-\frac{\omega^{2}}{4} L C}+j \frac{L}{2} \omega= \pm R_{K 1}+j X_{K}
$$

Here, $L$ and $C$ are the reactances of the coil and capacitor, respectively. Because their impedances are purely imaginary, the impedance of a circuit comprising finite numbers of coils and capacitors is also purely imaginary. However, a real part given by taking the square root is obtained in a periodic structure using a countable infinite number of elements and is denoted as $R_{K 1}$. This corresponds to resistance and means that active power is obtained and can be transmitted. However, the real part was obtained from the square of the impedance using the periodicity, and it may be a negative real part. If the impedance with a negative real part is obtained, amplification, an important phenomenon, is possible. In the following section, we will confirm this by using cascade matrices.

\subsection{Cascade matrices and iterative parameters}

The cascade matrix of the basic LC ladder circuit shown in Fig. 22.1(a) was expressed by Eq. (5.13) in Session 5 and is again given below.

$$
\left(\begin{array}{ll}
A & B \\
C & D
\end{array}\right)=\left(\begin{array}{cc}
1-\omega^{2} L C & j \omega L \\
j \omega C & 1
\end{array}\right)
$$

The iterative parameters of the LC ladder circuit were also shown in Session 5. Among them, the iterative phase $\beta_{a}$ was given by

$$
\cos \beta_{a}=\frac{2-L C \omega^{2}}{2}
$$

Waves with an angular frequency lower than $\omega_{c}$ are in the passband. For the waves in the passband, $\beta_{a}$ and two iterative impedances $Z_{J 1}$ and $Z_{J 2}$ are obtained, as shown in Fig. 22.2.

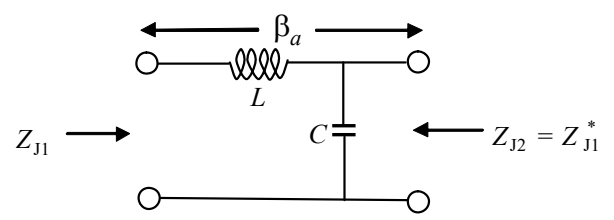

Fig.22.2 Iterative parameters of the circuit shown in Fig. 22.1(a)

Here, $\beta_{a}$ is given above, whereas $Z_{J 1}$ and $Z_{J 2}$ are respectively given by

$$
\begin{aligned}
& Z_{J 1}=\sqrt{\frac{L}{C}} \sqrt{1-\frac{\omega^{2}}{4} L C}+j \frac{L}{2} \omega=R_{K 1}+j X_{K} \\
& Z_{J 2}=R_{K 1}-j X_{K}=Z_{J 1} *
\end{aligned}
$$

The impedance given using squares, $Z$, shown in the previous section is obtained as $Z_{J 1}$ and $Z_{J 2}$ in Eqs. (22.8a) and $(22.8 \mathrm{~b})$, respectively, when the cascade matrix is used. They are the iterative impedances at the input and output terminals, as shown in Fig. 22.2, and are complex conjugates with the same positive real part, showing that impedance matching is realized. 


\subsection{LC ladder circuits}

Electric and magnetic fields were described by the Maxwell equations, from which the field theory was developed. Considering the Maxwell equations from the viewpoint of circuit theory, electric and magnetic fields correspond to the circuit elements called capacitors (charges) and coils, respectively. Thus, the field theory can be discussed on the basis of circuit theory, and we continue the discussion below.

In the previous section, we examined the iterative parameters using a countable infinite number of circuit elements. Here, we consider the use of a finite number of circuit elements and examine the power transmission characteristics of circuits with different combinations of the elements.

In Ref. [4], the power transmission characteristics of circuits are obtained using iterative and image parameters basically for four segments of the basic LC ladder circuit shown in Fig. 22.1(a). In Session 5, we used four segments of the basic LC ladder circuit in Ref. [4] and showed a circuit in Fig. 5.4 as an example using iterative parameters. Now, we refer to this example; namely, assuming that the internal resistance of a power source $R_{G}$ and the resistance of a load $R_{R}$ are equal and denoted as $R_{a}$, we calculate the power for different $R_{a}$ values of 5, 1 , and 0.1 , and show the power transmission characteristics. Note that $L=1$ and $C=$ 1 for simplicity.

(A) Asymmetric-periodic circuit

We pick the example shown in Fig. 5.4 in Session 5, which is shown again in Fig. 22.3. Here, the cascade matrix of a transmission circuit (a circuit in a black box) is given by

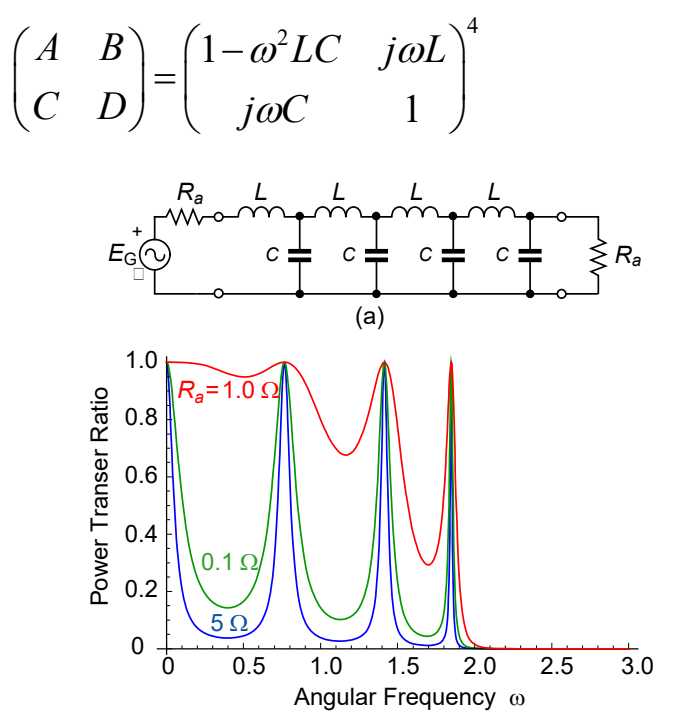

(b)

Fig.22.3 (a) Structure and (b) Power transmission characteristics of an asymmetric circuit with a periodic structure

(B) Symmetric-nonperiodic circuit
When a series element with inductance $L$ is connected to the right end of the transmission circuit shown in Fig. 22.3(a), a symmetric-nonperiodic circuit is obtained, as shown in Fig. 22.4(a).

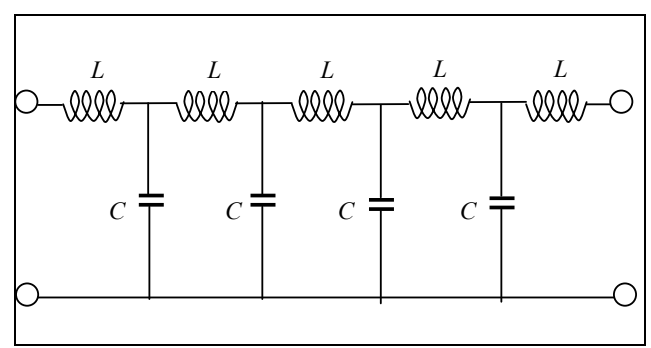

(a)

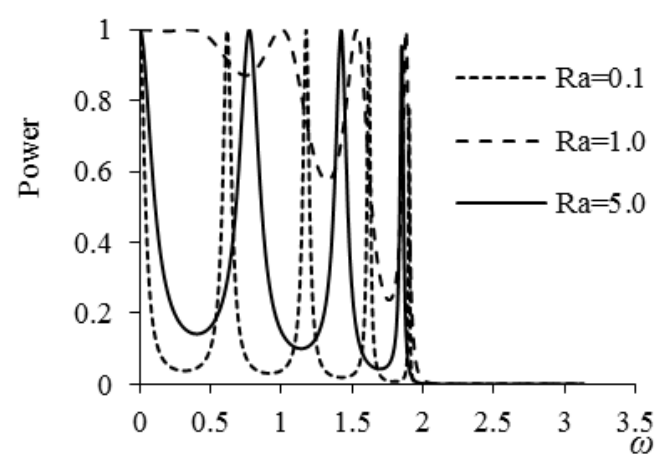

(b)

Fig.22.4 (a) A symmetric-aperiodic circuit and (b) its power transmission characteristic

We determine the power transmission characteristics of this circuit and determine by numerical calculation whether resonance is achieved. Figure 22.4(b) shows the power transmission characteristics. The cascade matrix of the circuit shown in Fig. 22.4(a) is given by

$$
\left(\begin{array}{ll}
A & B \\
C & D
\end{array}\right)=\left(\begin{array}{cc}
1-\omega^{2} L C & j \omega L \\
j \omega C & 1
\end{array}\right)^{4} \cdot\left(\begin{array}{cc}
1 & j \omega L \\
0 & 1
\end{array}\right)
$$

This indicates that the circuit is nonperiodic.

(C) Symmetric-periodic circuit

The basic segment of the circuit shown in Fig. 22.5(a) is expressed using image parameters. The circuit is symmetric, and its cascade matrix is given by

$$
\begin{aligned}
& \left(\begin{array}{cc}
1 & j \omega L / 2 \\
0 & 1
\end{array}\right) \cdot\left(\begin{array}{cc}
1 & 0 \\
j \omega C & 1
\end{array}\right) \cdot\left(\begin{array}{cc}
1 & j \omega L / 2 \\
0 & 1
\end{array}\right)= \\
& \left(\begin{array}{cc}
1-\omega^{2} \frac{L C}{2} & j \omega L-j \omega^{3} \frac{L^{2} C}{4} \\
j \omega C & 1-\omega^{2} \frac{L C}{2}
\end{array}\right)
\end{aligned}
$$

In the passband, Eq. (22.11) is rewritten using image parameters as 


$$
\left(\begin{array}{ll}
A & B \\
C & D
\end{array}\right)=\left(\begin{array}{cc}
\cos \beta_{c} & j R_{K 1} \sin \beta_{c} \\
\frac{j}{R_{K 1}} \sin \beta_{c} & \cos \beta_{c}
\end{array}\right)
$$

Here,

$$
\begin{aligned}
& R_{K 1}=\sqrt{\frac{L}{C}} \sqrt{1-\frac{L C}{4} \omega^{2}} \\
& \cos \beta_{c}=1-\omega^{2} \frac{L C}{2} \\
& \sin \beta_{c}=\omega \sqrt{L C} \sqrt{\left(1-\omega^{2} \frac{L C}{4}\right)}
\end{aligned}
$$

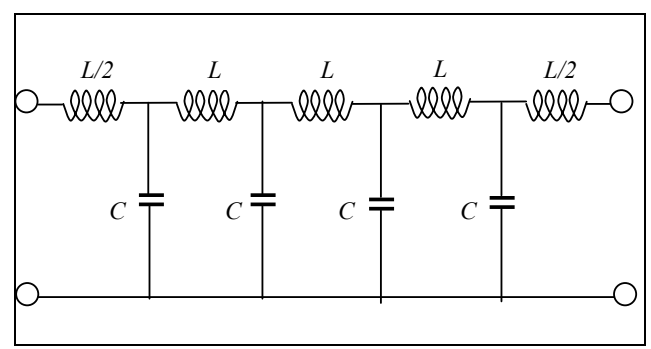

(a)

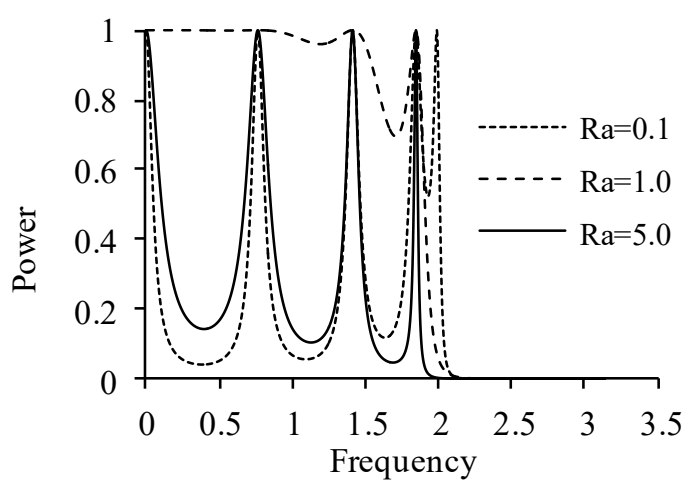

(b)

Fig.22.5 (a) A symmetric-periodic circuit and (b) its power transmission characteristic

Thus, the cascade matrix of the circuit shown in Fig. 22.5 (a) can be expressed using image parameters. Note that the cascade connection of basic segments is expressed by the product of the cascade matrices of the basic segments when image parameters are used. In the circuit shown in Fig. 22.5(a), four basic segments with the cascade matrix given by Eq. (22.12a) are cascade-connected. The cascade matrix of this circuit in the passband is given by

$$
\left(\begin{array}{cc}
\cos \beta_{c} & j R_{K 1} \sin \beta_{c} \\
\frac{j}{R_{K 1}} \sin \beta_{c} & \cos \beta_{c}
\end{array}\right)^{4}=\left(\begin{array}{cc}
\cos 4 \beta_{c} & j R_{K 1} \sin 4 \beta_{c} \\
\frac{j}{R_{K 1}} \sin 4 \beta_{c} & \cos 4 \beta_{c}
\end{array}\right)
$$

When a load with a resistance of $R_{a}$ is connected to a circuit with this cascade matrix, the input impedance $Z_{i n}$ is given by

$$
Z_{\text {in }}=\frac{R_{K 1} R_{a} \cos 4 \beta_{c}+j R_{K 1}^{2} \sin 4 \beta_{c}}{j R_{a} \sin 4 \beta_{c}+R_{K 1} \cos 4 \beta_{c}}
$$

If impedance matching occurs in the above input impedance and internal resistance $R_{a}$ of the source, resonance is generated. Therefore, by assuming that Eq.(22.14) and $R_{a}$ are equal, and the real part and imaginary part are equal, the following equation of imaginary part can be obtained.

$$
R_{a}^{2} \sin 4 \beta_{c}=R_{K 1}^{2} \sin 4 \beta_{c}
$$

For the above equation to hold,

$$
R_{a}=R_{K 1}
$$

or

$$
\sin 4 \beta_{c}=0
$$

We can recognize resonance for which Eq. (22.16a) holds because, in Fig. 22.5(b), there is a point of resonance at $R_{a}=0.1$ and the place where the value of abscissa is extremely close to 2. As for resonance for which Eq. (22.16b) holds, image phase $\beta_{c}$ within the passband is between $0^{\circ}$ and $180^{\circ}$, so the valid range of $4 \beta_{c}$ is

$$
0 \leqq 4 \beta_{c}<4 \pi
$$

Within the above range, the number of $4 \beta_{c}$ satisfying Eq.(22.16b) is four as follows.

$$
4 \beta_{c}=0, \pi, 2 \pi, 3 \pi
$$

If these four values are converted into angles,

$$
\beta_{c}=0^{\circ}, 45^{\circ}, 90^{\circ}, 135^{\circ}
$$

Furthermore, if we assume $R_{a}=0$, it becomes the eigenvalue problem. Eigenvalues appear at five points. If $R_{a}$ $=\infty$, that is, the two terminals are open, eigenvalue appears too, and a point spectrum is obtained at four points.

As this circuit is a symmetrical circuit and image resistance is a real number, it is possible that characteristic vibration is obtained when internal resistance and load resistance are 0 and $\infty$, but we leave this problem unsolved.

The features of the circuit determined using image parameters are described in detail in Ref. [4] and are not explained here.

Figure 22.5(b) shows the power transmission characteristics of the circuit shown in Fig. 22.5(a).

\subsection{Resonnant phases generated by periodic structure}

Regarding the generation of waves and oscillation, Ref. [3] introduces a simple harmonic oscillation, or a harmonic oscillation, which is expressed using a series LC resonant circuit. Waveforms of different angular frequencies can be produced in coupled oscillation systems, which are related to Fourier series. In the transmission circuits with periodic structures (A) and (C) described in Section 2.3, the resonance angular frequency is unchanged even if the resistance of the input and output ports is changed, meaning 
that the resonance frequency of waves generated in the periodic circuits is unchanged. Therefore, it is shown that waves and oscillations with the phase $\mathrm{k} \pi(\mathrm{k}=1,2, \cdots, \mathrm{n}-1)$ of the resonance angular frequency are obtained in periodic circuits. The generation of waves with the resonance angular frequency in periodic LC ladder circuits is different from that in coupled oscillation systems and is considered to be a physical phenomenon obtained in circuit theory.

As explained above, cascade matrices are essential for circuit theory. Reciprocity and lossless properties of circuits in the steady state are shown by the cascade matrices, which also enable the discrimination of active and reactive powers. Moreover, circuit analysis and synthesis [5] are performed using cascade matrices.

\section{Maxwell Equations by Vector Analysis}

The purpose of this lecture series is to apply circuit theory to quantum mechanics. The origin of quantum mechanics is said to be the discovery of the Maxwell equations [1]. Hence, we examine the vector analysis $[3,6,7]$ that is used for analyzing the Maxwell and Schrödinger equations in physics and compare it with the analytical method based on circuit theory.

In an introductory textbook of vector analysis [6], Soda explains the Lagrange equation and Hamilton's principle as follows: 'Hamilton's principle is a very compact and beautiful formulation in classic mechanics. However, the most important point of this principle is that phenomena in classical electromagnetism can be formulated by applying the same variational principle to an arbitrary Lagrange function and that phenomena in quantum mechanics can also be formulated by extending this principle.' It is also argued that the Maxwell equations and quantum mechanics were systemized by the Lagrange equation and Hamilton's principle.

In Ref. [7], Kitano stated: 'The law of conservation of electromagnetic fields gives the equation of relating squared quantities in a field. For example, quantities such as $E_{i} E_{i}$ and $B_{i} H_{j}$ are introduced. These squared quantities cause many problems that are difficult to interpret in wave phenomena, particularly the interference of waves, and the particle and wave natures conflict. In this sense, the law of conservation of electromagnetic fields is a treasure box of paradoxes.' This seems to suggest that the law of conservation of energy may not hold in some cases even though the Maxwell equations use a Hamiltonian based on the law of conservation of kinetic energy.

In Ref. [8], Takahashi, who is familiar with circuit theory, pointed out: "The principle of least action that gives the equation of motion of a single mass is very similar to the equation called Fermat's principle in geometry but the velocity $v$ appears in the numerator for the former and in the denominator for the latter. This difference is related to the previous dispute between the particle and wave theories of light.'

From the above example, the Hamiltonian appropriately represents the law of conservation of energy in Newtonian mechanics but may not appropriately represent the law of conservation of energy for electromagnetic waves in some cases. With this assumption, we examine the Hamiltonian in more detail.

In Ref. [3], Sato examined an example of geometrical optics. The path of light is straight when the refractive index of a medium is constant regardless of position. However, when the refractive index changes as a function of position, he stated: "The path of light traveling between points $\mathrm{A}$ and $\mathrm{B}$ is the path that minimizes the time taken by the light to reach point $\mathrm{B}$ from point A, according to Fermat's principle."

Although the path of light is determined according to Fermat's principle, the variational principle cannot be applied to light or electromagnetic waves. When circuit theory is applied to light and electromagnetic waves, the law of conservation of energy is not satisfied as a steadystate response although the path can be determined as a transient response. Namely, the law of conservation of energy is violated unless the energy of reflected waves is added to that of light in the path. Because a refracted wave means a transmitted wave and generates a reflected wave according to the principle of the transmission and reflection of waves, the energy of the reflected wave should be considered to satisfy the law of conservation of energy.

An example that requires the application of circuit theory is "partial reflection of light at two surfaces of a thin glass" as shown in Ref. [9]. The refraction and reflection of light can explain why soap bubbles are colored and why peacocks and hummingbirds have such a vivid color. Note that the idea of distributed constant circuits originates from the desire to design circuits for filters using transmission lines with different refractive indices and that commensurate transmission line circuits [10] were a theoretical answer.

In this session, we showed that the law of conservation of energy is not satisfied because of the generation of reflected waves when a Hamiltonian is used in the analysis of the Maxwell equations. Therefore, analysis using the Hamiltonian does not consider the law of conservation of energy also for the wave nature in quantum mechanics, which will require some time for us to reexamine.

Prof. Sanada at Hokkaido University of Science and Prof. Motoki at Hokkai-Gakuen University will write articles related to this lecture series as Sessions 23 and 24, respectively.

\section{Acknowledgments}

We are deeply grateful to Professor Kazuhisa Watanabe at Hokkaido University of Science for their continued cooperation in our discussion. We are deeply grateful to 
Professor Hiroshi Tanimoto at Kitami Institute of Technology for his continued cooperation in our discussion via e-mail. We also thank Dr. Jie Ren for her continued support in the development of the figures and calculations.

\section{References}

[1] N. Forbes and B. Mahon: FARADAY, MAXWELL, AND ELECTROMAGNETIC FIELD, How Two Men Revolutionized Physics, Prometheus Books, Inc., 2014.

[2] M. Igawa: Hyperbolic Partial Differential Equations and Wave Phenomena, Iwanami Shoten, 2006. (in Japanese)

[3] H. Sato: Physical Mathematics Note (To Better Understand Basic Physics), Separate Issue of Mathematical Sciences: SGC Library 20, Saiensusha Co., Ltd., 2003. (in Japanese)

[4] N. Nagai: Circuit Theory Based on New Concept and Its Application to Quantum Mechanics, Seibunsha, 2013. (in Japanese)

[5] H. Watanabe: Theory and Design of Transmission Networks, Ohmsha, Ltd., 1968. (in Japanese)

[6] J. Soda: Analytical Mechanics for Modern Physics, Separate Issue of Mathematical Sciences, SGC Library 46, Saiensusha Co., Ltd.., 2006. (in Japanese)

[7] M. Kitano: Maxwell Equations (To Better Understand Electromagnetics) SGC Library 39, Saiensusha Co., Ltd., 2005. (in Japanese)

[8] H. Takahashi and O. Fujimura: The Hidetoshi Takahashi Lectures on Physics - General Theory, Maruzen Co., Ltd., 1990. (in Japanese)

[9] R. P. Feynman: QED - The Strange Theory of Light and Matter -, Princeton University Press, 1985.

[10] A. Matsumoto, Ed.: Microwave Filters and Circuits, Academic Press, 1979.

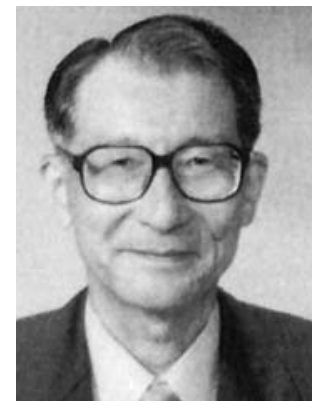

Nobuo Nagai received his B.S. and D.Eng. degrees from Hokkaido University in 1961 and 1971, respectively. In 1961, he joined Hokkaido University as an Assistant and in 1972 he became an Associate Professor, and from 1980 to 1992 he was a Professor in the Research Institute of Applied Electricity. From 1992 to 2001, he was a Professor in the Research Institute for Electronic Science, Hokkaido Uni-

University. In 2001, he retired and became an Emeritus Professor. His research interests are circuit theory and digital signal processing. He is interested in the application of above theory to quantum theory. Dr. Nagai is a Life Fellow of the Institute of Electronics, Information and Communication Engineers, Japan, and a Life Member of IEEE and IEICE, and an Honorary Member of RISP.

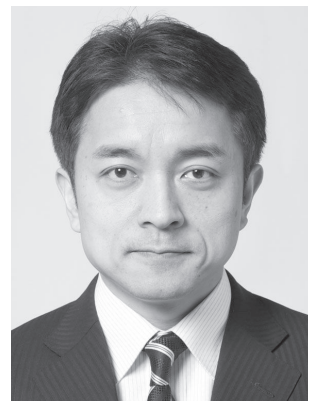

Hirofumi Sanada received his B.S. and Ph.D. degrees from Hokkaido University in 1991 and 1998, respectively. In 1994, he joined Hokkaido University as a Research Associate and in 2002, he became an Instructor in Hokkaido Univversity of Science (HUS). Since 2014 he has been a Professor in HUS. His research interests are circuit theory, numerical simulation of wave propagation phenomena. He is also interested in the application of above theory to quantum theory. Prof. Sanada is a member of IEICE and IEEE.

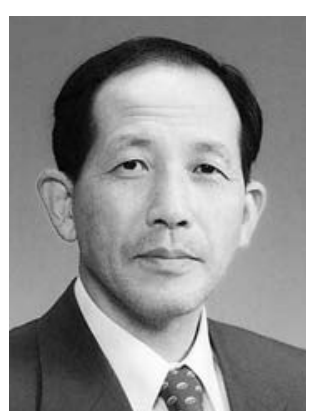

Takashi Yahagi received his B.E., M.S. and Ph.D. degrees all from the Tokyo Institute of Technology in 1966, 1968 and 1971, respectively. In 1971, he joined Chiba University as a Lecturer and in 1974 he became an Associate Professor, and from 1984 to 2008 he was a Professor at the same university. Since 2008 he has been with the Signal Processing Research Laboratory. In 1997, he founded the Research Institute of Signal Processing, Japan (RISP). Since 1997 he has been President of RISP. From 1997 to 2013 he was Editor-in-Chief of the Journal of Signal Processing (JSP). Since 2013 he has been Honorary Editor-in-Chief of JSP. He was the author of "Theory of Digital Signal Processing (Vols. 1-3)", (1985, 1985, 1986), Corona Pub.Co., Ltd. (Tokyo, Japan). He was also the editor and author of "Library of Digital Signal Processing (Vols. 1-10)", (1996, 2001, 1996, 2000, 2005, 2008, 1997, 1999, 1998, 1997), Corona Pub.Co., Ltd. (Tokyo, Japan). He was the editor of "My Research History (Vols. 1 and 2)” (2003, 2003), RISP. The contents of the Library of Digital Signal Processing are as follows: Vol.1: Digital Signal Processing and Basic Theory (1996), Vol.2: Digital Filters and Signal Processing (2001), Vol.3: Digital Signal Processing of Speech and Images (1996), Vol.4: Fast Algorithms and Parallel Signal Processing (2000), Vol.5: Kalman Filter and Adaptive Signal Processing (2005), Vol.6: ARMA Systems and Digital Signal Processing (2008), Vol.7: VLSI and Digital Signal Processing (1997), Vol.8: Communications and Digital Signal Processing (1999), Vol.9: Neural Network and Fuzzy Signal Processing (1998), Vol.10: Multimedia and Digital Signal Processing (1997). Dr. Yahagi is a Life Fellow of the Institute of Electronics, Information and Communication Engineers, Japan. 